\title{
Pushing the boundaries of the management of uncomplicated diverticulitis
}

\author{
Y. Ribas $^{1}$ - A. D'Hoore ${ }^{2}$
}

Received: 9 March 2016/Accepted: 10 March 2016/Published online: 29 March 2016

(C) Springer-Verlag Italia Srl 2016

The incidence of diverticulitis has increased worldwide and poses a significant burden on healthcare resources. Fortunately, most cases of acute diverticulitis are uncomplicated and may be treated conservatively. The literature on acute diverticulitis has increased substantially, leading to a reconsideration of the conventional wisdom, which was mostly based on surgeons' personal preferences. Nevertheless, some aspects of the traditional approach may be easier to change than others. Many doctors may have wondered about the rationale for dietary restrictions or the need for hospital admission of patients with uncomplicated diverticulitis. However, other issues such as the need for antibiotics are still controversial, and the support of robust evidence will be required to change common practice. It should be emphasized that, although recent publications have challenged the accepted dogmas, recent guidelines remind us that the quality of evidence for most management issues is low.

It is internationally accepted that most patients with uncomplicated diverticulitis may be safely managed on an outpatient basis if they can tolerate oral intake, pain control is achieved, and social support is effective [1]. Adequate case selection depends, however, on an accurate diagnosis, and there is a general agreement on the need for a computed tomography (CT) scan to confirm the clinical suspicion and to distinguish between uncomplicated and complicated diverticulitis. Ultrasound has a high sensitivity

Y. Ribas

yribas@cst.cat

1 Department of Surgery, Consorci Sanitari de Terrassa, Terrassa, Barcelona, Spain

2 Department of Abdominal Surgery, University Hospital Gasthuisberg, Leuven, Belgium and specificity in the hands of experienced operators, and some authors have suggested that CT should only be performed after a negative or inconclusive ultrasound.

Regarding diet during the acute episode, conservative treatment included bowel rest or dietary restrictions, but there is no evidence that these practices influence the outcome of acute diverticulitis. Moreover, the literature on outpatient management of uncomplicated diverticulitis usually recommends a liquid diet for some days followed by a low-fiber diet. However, there is no rationale for this approach either. The authors' standard practice is to prescribe a regular diet.

Antibiotics have been the cornerstone of the treatment of acute diverticulitis, based on the assumption that it had an infectious basis. However, recent studies support the theory that it may be an inflammatory, rather than infectious condition, thus challenging the unquestionable need for antibiotics. The routine use of antibiotics has been questioned in a large randomized study involving more than 600 patients with acute uncomplicated diverticulitis confirmed with a CT scan, who were admitted to 11 centers (10 in Sweden and 1 in Iceland) [2]. Exclusion criteria included signs of complicated diverticulitis on CT as well as sepsis or peritonitis, which were not clearly defined. Eligible patients randomized to the antibiotic group were treated with broad-spectrum antibiotics covering gramnegative and anaerobic bacteria, according to the protocol in each participating center, for 7 days. The nonantibiotic group only received intravenous fluids. Information on diet and other medication is not provided. Ten patients (3.2\%) allocated to the nonantibiotic group required antibiotics, and there were no differences between groups regarding morbidity or the need for surgical treatment. The authors concluded that antibiotics did not accelerate recovery nor prevent complications or recurrence. Some recent 
guidelines already recommend a rather selective and individualized use of antibiotics based on this single study [36], although the American Gastroenterological Association technical review rated the quality of this evidence as low, because of risk of bias and imprecision [7]. Another study from the same investigators prospectively assessed outpatient management without antibiotics in patients with CTconfirmed uncomplicated diverticulitis [8]. In this study, 155 patients were discharged from the emergency department and were recommended only fluids for $48 \mathrm{~h}$ followed by a liquid diet progressing to a normal diet, and paracetamol. Only 4 patients $(2.6 \%)$ were admitted due to worsening of symptoms, and all of them were successfully managed with antibiotics. A limitation of this study is that 66 patients who fit inclusion criteria during the study time period were not included, which represents a selection bias. In neither of the two studies mentioned above, it is clear what percentage of patients with diverticulitis was eligible for the study. In the current issue, Estrada et al. [9] report on the selective use of antibiotics in patients with CTconfirmed Neff 0 (thickening of the wall, increased density of the pericolic fat) sigmoid diverticulitis. These Neff 0 diverticulitis patients represented $50 \%$ of the patients seen in the emergency department in the study period. Forty-five patients with uncomplicated diverticulitis were managed without antibiotics. Of them, 36 were managed as outpatients, only $4(11 \%)$ requiring admission (3 of them successfully treated without antibiotics). The remaining 9 were admitted, and treated without antibiotics, but 3 of them were subsequently readmitted. Overall, $93 \%$ of patients were managed without antibiotics, and none of the patients presented complications or required surgery. Few papers on this topic have been published so far, but they have concluded that nonantibiotic management is safe and effective in selected cases, with low readmission and complication rates. Although the complication rate in these studies is very low, the authors' opinion is that the results of these studies should not be generalized to all patients with uncomplicated diverticulitis and we have continued to prescribe oral antibiotics to patients with mild diverticulitis until more evidence is available. Further trials and results of ongoing studies are eagerly awaited to identify those patients in whom antibiotics may not be required. In this respect, the DIABOLO trial [10], an ongoing randomized controlled trial currently recruiting patients in the Netherlands, might confirm this new evidence.

Based on the assumption that underlying chronic inflammation may be responsible for recurrence after an acute episode, some authors have suggested the use of different treatments, including nonabsorbable antibiotics, anti-inflammatory drugs, and probiotics. However, an extensive review from the American Gastroenterological Association recommended against the use of mesalamine, rifaximin, and probiotics. While mesalamine was not shown to be effective enough, rifaximin and probiotics were not recommended due to the very low quality of evidence [6]. Therefore, high-quality studies are needed to assess the true efficacy of these agents.

A better understanding of the pathophysiology and natural history of diverticular disease has led to the reconsideration of a myriad of aspects of the management of diverticulitis and to a more conservative approach among surgeons. As research progresses, clinical practice should follow.

\section{Compliance with ethical standards}

Conflict of interest None.

\section{References}

1. Biondo S, Golda T, Kreisler E et al (2014) Outpatient versus hospitalization management for uncomplicated diverticulitis: a prospective, multicenter randomized clinical trial (DIVER trial). Ann Surg 259:38-44

2. Chabok A, Pahlman L, Hjern F, Haapaniemi S, Smedh K (2012) Randomized clinical trial of antibiotics in acute uncomplicated diverticulitis. Br J Surg 99:532-539

3. Andersen JC, Bundgaard L, Elbrond H, Laurberg S, Walker LR, Stovring J (2012) Danish national guidelines for treatment of diverticular disease. Dan Med J 59:C4453

4. Andeweg CS, Mulder IM, Felt-Bersma RJ, Netherlands Society of Surgery; Working group from Netherlands Societies of Internal Medicine, Gastroenterologists, Radiology, Health technology Assessment and Dieticians et al (2013) Guidelines of diagnostics and treatment of acute left-sided colonic diverticulitis. Dig Surg 30:278-292

5. Kruis W, Germer CT, Leifeld L, German Society for Gastroenterology D, Metabolic D, The German Society for General and Visceral Surgery (2014) Diverticular disease: guidelines of the german society for gastroenterology, digestive and metabolic diseases and the german society for general and visceral surgery. Digestion 90:190-207

6. Stollman N, Smalley W, Hirano I, AGA Institute Clinical Guidelines Committee (2015) American Gastroenterological Association Institute guideline on the management of acute diverticulitis. Gastroenterology 149:1944-1949

7. Strate LL, Peery AF, Neumann I (2015) American Gastroenterological Association Institute Technical review on the management of acute diverticulitis. Gastroenterology 149(19501976):e12

8. Isacson D, Thorisson A, Andreasson K, Nikberg M, Smedh K, Chabok A (2015) Outpatient, non-antibiotic management in acute uncomplicated diverticulitis: a prospective study. Int J Colorectal Dis 30:1229-1234

9. Estrada-Ferrer O, Hidalgo-Grau LA, Abadal-Prades $M$ et al (2016) Selective outpatient treatment without antibiotic in sigmoid diverticulitis. Is it time to change traditional practices? Tech Coloproctol 2016 (in press)

10. Unlu C, de Korte N, Daniels L, Dutch Diverticular Disease 3D Collaborative Study Group et al (2010) A multicenter randomized clinical trial investigating the cost-effectiveness of treatment strategies with or without antibiotics for uncomplicated acute diverticulitis (DIABOLO trial). BMC Surg 20:23 